\title{
Du conteur au narrateur
}

L'imaginaire de la veillée chez Nikolaï Gogol et George Sand

\section{Victoire Feuillebois}

\section{(2) OpenEdition}

Journals

Édition électronique

URL : https://journals.openedition.org/clo/1894

DOI : $10.4000 /$ clo. 1894

ISSN : 2266-1816

Éditeur

INALCO

Édition imprimée

Date de publication : 1 janvier 2014

ISBN : 978-2-85831-222-1

ISSN : 0396-891X

Référence électronique

Victoire Feuillebois, « Du conteur au narrateur », Cahiers de littérature orale [En ligne], 75-76 | 2014, mis en ligne le 29 avril 2015, consulté le 01 juillet 2021. URL : http://journals.openedition.org/clo/1894 ; DOI : https://doi.org/10.4000/clo.1894

Ce document a été généré automatiquement le 1 juillet 2021.

\section{(c) (i) (2)}

Cahiers de littérature orale est mis à disposition selon les termes de la Licence Creative Commons Attribution - Pas d'Utilisation Commerciale 4.0 International. 


\title{
Du conteur au narrateur
}

\author{
L'imaginaire de la veillée chez Nikolaï Gogol et George Sand
}

\author{
Victoire Feuillebois
}

Même lorsqu'ils s'écrivent en pleine "galaxie Gutenberg", les textes peuvent entretenir des rapports avec le régime de la littérature orale : c'est particulièrement le cas dans la sensibilité littéraire romantique, qui refuse en général de disqualifier l'oralité pure comme une forme brute d'expression infralittéraire, mais l'érige au contraire en modèle poétique privilégié, proche de l'enfance et de la nature, et à ce titre susceptible de donner accès à la langue originelle (Ursprache) désignée dès 1771 par le philosophe Herder comme l'horizon de toute production artistique. Ainsi, on ne s'étonnera pas de trouver des figures de conteurs paysans à la source des œuvres que nous proposons d'étudier et qui appartiennent au canon de la littérature romantique, les Veillées du hameau (1830-1843) de Nikolaï Gogol et les Veillées du chanvreur (1844-1849) de George Sand. Pourtant, s'il existe chez les deux auteurs une fascination objective pour le phénomène réel du contage, cet article se penche sur une autre valeur de l'imaginaire du récit paysan: selon nous, l'oralité figurée dans les textes n'est pas seulement un thème de l'œuvre, un élément de décor ou d'atmosphère, mais elle engage l'organisation des instances narratives dans le texte en participant à la constitution d'une figure d'autorité narrative par un jeu d'associations et de dissociations entre le conteur représenté et le narrateur effectif du récit. En effet, à la manière du «discours d'assistance " repéré par Sarah Kofman au sujet de l'écrivain romantique E.T.A. Hoffmann (Kofman, 1984), les auteurs étudiés semblent s'appuyer sur un «encadrement» de leur discours par des étais extérieurs, constitués ici par la représentation de la veillée paysanne avec ses conteurs hauts en couleur et ses récits insérés pittoresques; mais cette structure n'est pas imitée jusqu'au bout, et finit par neutraliser l'assimilation, laissant deviner la maîtrise du narrateur au-delà du masque du conteur. C'est d'autant plus important que ces œuvres qui s'inspirent de la veillée paysanne dans leur structure narrative ont souvent été l'objet de deux reproches de la part des critiques littéraires : par rapport à la narration plus homogène et détachée du roman moderne, le dénivelé narratif qu'elles adoptent (avec un premier cadre décrivant les circonstances de la veillée et des récits insérés faits par le conteur du cadre) et la crédibilisation externe du texte (qui se présente comme la transcription 
d'une soirée à la campagne et paraît présenter le contage comme la source réelle du texte) semblent les rejeter dans une forme archaïque de récit. Or, adopter cet autre point de vue sur l'inscription des figures de conteur dans le texte littéraire revient à esquiver ces reproches : il ne s'agit ni d'établir une différence essentielle entre oralité et écriture, ni de déterminer la part des sources orales dans la production écrite. Notre objectif est ici de souligner que l'imaginaire de la veillée dans nos textes ne se réduit pas à une nostalgie pour les formes directes de communication littéraire encore en cours à la campagne: la projection de l'organisation narrative du texte sur l'organisation réelle d'une veillée paysanne constitue un mode particulier de représentation auctoriale, reposant sur une dissociation entre le conteur, tout entier absorbé par une oralité rurale qui légitime le statut documentaire de la fiction, et le narrateur qui, lui, s'affirme progressivement comme une figure suprême de contrôle sur le récit et l'agent de l'harmonisation ultime de ses strates. Ainsi, l'inclusion du récit oral imité de la veillée paysanne a un double bénéfice : elle est le garant de la véridicité et du pittoresque de l'œuvre, mais elle répond à la nécessité narrative de mettre en relief la figure discrète du narrateur, qui se constitue par le jeu avec cette « autre voix de la littérature écrite ».

\section{Le conteur, support d'autorité charismatique pour le récit ou facteur de déséquilibre de la narration?}

2 Toute une tradition critique a souligné l'intérêt de la littérature romantique pour la matière folklorique et les effets d'oralisation, qui apparaissent comme les manières d'appuyer la parole du "mage romantique» (Bénichou, 1988) et de lutter contre «le désenchantement du monde", pour reprendre la formule de Max Weber (2005) empruntée au poète allemand Friedrich Schiller (1992, 194, v. 168). Mais cette approche de l'oralité dans les textes romantiques a été depuis critiquée par des théoriciens qui, dans la lignée du formalisme ou de la déconstruction, remettent en cause l'idée que la littérature puisse s'inspirer d'autre chose qu'elle-même : de la lettre à la parole, n'y a-til pas une fausse continuité, une illusion de présence qui brouille l'horizon essentiellement textuel de toute écriture? Entre ces deux postures opposées est apparue récemment une troisième approche du problème de l'oralité dans la littérature romantique: inspirée de l'histoire culturelle, celle-ci s'appuie sur la notion de "figurations auctoriales» (Diaz, 2007) pour interroger, non pas la présence effective d'une matière orale identifiable dans le texte, mais les investissements imaginaires que les auteurs romantiques font de l'oralité, dans un contexte de technologisation et de mercantilisation grandissantes des lettres (Vaillant, 2005, 2010). Les deux textes étudiés ici mettent en valeur ce double intérêt de l'inscription orale dans le texte écrit: le modèle narratif de la veillée paysanne permet à la fois de profiter de l'attrait pour la parole vive typique de la sensibilité romantique et de faire ressortir par contraste la figure d'un narrateur détaché de l'horizon de la veillée elle-même.

3 "J'ai commencé, par la Mare au Diable, une série de romans champêtres, que je me proposais de réunir sous le titre de Veillées du Chanvreur » (Sand, 1997, t. 1, 164) : dans la notice rédigée pour la publication de ses œuvres complètes chez Hetzel en 1851, George Sand évoque son projet d'écrire un cycle de Veillées du chanvreur, qui regroupe en définitive trois de ses œuvres les plus célèbres, la Mare au Diable (1846), François le Champi (1848) et la Petite Fadette (1849), unifiées par la figure du chanvreur. 
Pourtant, l'apparent retrait derrière ce personnage dissimule en réalité une stratégie littéraire élaborée : ces textes s'inscrivent dans le schéma traditionnel de l'autorité littéraire, fondée sur l'appui de sources extérieures et sur l'exhibition de voix narratives distinctes de celle de l'auteur, plus pittoresques et mieux aptes à justifier l'existence du texte - mais c'est pour mieux en subvertir le fonctionnement et faire ressortir l'activité du narrateur. La dissociation des instances orales et écrites permet en effet un glissement qui mène du « chanvreur » dont la narratrice sandienne prétend n'être que la chambre d'enregistrement, au « chantre du Berry » (Lubin, 1992) : dans les romans champêtres, du cycle du chanvreur aux Maîtres-sonneurs (1853) qui reprennent une organisation en « veilles ", la " présence de la voix » est une amorce de la « venue à l'écriture » (Beizer, 2004).

4 Mais cette voix pittoresque apparaitt bien d'abord comme un élément fondamental pour légitimer le texte et lui conférer de l'intérêt. Le chanvreur qui unifie les différents romans entre eux et garantit l'unité du cycle est une figure de conteur nocturne largement anonyme, incarnant une réalité socioculturelle que Sand présente comme le point focal et la justification de son cycle, la veillée :

Le rôle que joue en Bretagne le bazvalan, le tailleur du village, c'est le broyeur de chanvre ou le cardeur de laine (deux professions souvent réunies en une seule), qui le remplit dans nos campagnes. Il est de toutes les solennités tristes ou gaies, parce qu'il est essentiellement érudit et beau diseur, et, dans ces occasions, il a toujours le soin de porter la parole pour accomplir dignement certaines formalités usitées de temps immémorial. Les professions errantes, qui introduisent l'homme au sein des familles sans lui permettre de se concentrer dans la sienne, sont propres à le rendre bavard, plaisant, conteur et chanteur.

Le broyeur de chanvre est particulièrement sceptique. Lui et un autre fonctionnaire rustique, dont nous parlerons tout à l'heure, le fossoyeur, sont toujours les esprits forts du lieu. Ils ont tant parlé de revenants et ils savent si bien tous les tours dont ces malins esprits sont capables, qu'ils ne les craignent guère. C'est particulièrement la nuit que tous, fossoyeurs, chanvreurs et revenants, exercent leur industrie. (Sand, 1964, 156).

5 Ce narrateur présent à l'intérieur de la diégèse est le dénominateur commun de toutes les histoires et il prête son nom au titre imaginé pour la trilogie : il raconte du début à la fin la Petite Fadette, intervient directement dans la Mare au diable, prend en charge toute la deuxième partie du récit dans François le Champi. Il prend aussi l'initiative de ces histoires, bien qu'il partage parfois la narration avec d'autres personnages de ses récits: la première partie de François le Champi est ainsi racontée par la servante du curé. Il a beau être " un paysan inculte » et ne pas être nommé dans les textes, l'accent est mis sur le caractère prenant de ses récits, car il est « heureusement fort éloquent à sa manière » (Sand, 1976, 52) ; la servante du curé est quant à elle " une paysanne un peu cultivée " (Ibid.), signe que ces personnages ont un don spontané du récit. Les "veillées $d u$ chanvreur " semblent donc s'interpréter comme un génitif subjectif, et faire de ce personnage la source et le garant des récits. Il représente la justification du récit par des auctoritates distinctes de l'auteur lui-même: dans le cadre d'un roman paysan, l'inculture du paysan et sa langue rugueuse sont même un signe d'authenticité, une nouvelle manière de justifier le récit en soulignant sa couleur locale et son caractère véridique.

6 Le conteur figuré joue ici un rôle fondamental pour légitimer l'existence du texte, à la fois parce que la fiction est adossée à une pratique réelle (le texte prend alors une valeur documentaire, l'année même où William Thoms avance le terme de «folklore ») 
et parce qu'il s'inscrit dans une parole directe à la nuit tombée qui lui confère, pour reprendre les termes de Max Weber, une aura charismatique. Mais on voit d'emblée que la place symbolique et narrative accordée au conteur risque d'empiéter sur le domaine de l'auteur : c'est d'autant plus vrai dans le cas d'un chanvreur dont l'activité de tissage (textere en latin) est liée symboliquement à la confection du texte. À l'inverse, l'auteur peut apparaître comme le garant du texte (c'est l'un des sens du terme latin auctor, qui peut aussi désigner le "témoin»), puisqu'il a été le témoin des veillées, il peut se présenter comme l'instance d'accroissement d'une matière textuelle qui lui préexiste (du latin augere, "augmenter »), mais il ne peut apparaître à première vue dans son sens contemporain, comme une figure professionnelle qui détient l'autorité sur son texte.

7 Le même paradoxe est à l'œuvre dans lesVeillées du hameau près de Dikanka (1831-1832). Ce texte de jeunesse de Gogol se développe à partir d'une situation d'énonciation qui mime l'oralité nocturne et présente un conteur exubérant, le jovial apiculteur Panko le Rouge, reliant entre eux dans le cadre les récits insérés. La prégnance de la veillée nocturne renvoie ici à l'ancrage magique du texte, qui, selon les analyses célèbres d'Andreï Siniavski (Tertz, 1978), signale le désir gogolien de se faire enchanteur et de projeter sur les processus de création littéraire, considérée à l'époque moderne comme une activité anodine, l'ancienne faculté magique d'animer l'inanimé et de mettre en mouvement un monde imaginaire, peuplé de créatures fantastiques.

La veillée permet d'abord de convoquer une matière folklorique et surnaturelle qui étaie la fonction d'enchantement conférée au texte. Le recours à la nuit est ici surdéterminé : outre l'image de la veillée paysanne incarnée par Panko, les histoires insérées convoquent une matière fantastique où le diable intervient sans cesse dans l'obscurité, sur le mode du vertep, le théâtre de marionnettes ukrainien qui mélange le surnaturel et le quotidien. Cette matière est particulièrement présente dans la première nouvelle du cycle, « la Foire de Sorotchintsy ». Le texte introduit le ton leste et joyeux propre à la narration de Panko le Rouge en développant deux intrigues parallèles: d'une part, le mariage de la belle Paracha et du rusé Gritsko, auquel s'oppose une malveillante belle-mère; d'autre part, l'énigme d'une mystérieuse casaque rouge, qu'un diable en goguette a naguère perdu à la foire de Sorotchintsy et dont il entreprend depuis plusieurs années de récupérer les morceaux épars - au moment où commence l'histoire, il ne lui reste plus qu'à reprendre possession de sa manche gauche, qui se retrouve de manière comique en possession de différents marchands à qui elle porte le guignon, tandis que le démon se manifeste sous la forme d'un cochon pour retrouver son bien. Ces deux histoires n'ont à l'origine pas le même statut : là où l'aventure des noces contrariées est rapportée sur le mode de l'événement, l'odyssée de la casaque, d'emblée fantastique, est d'abord présentée comme une rumeur avant que les protagonistes ne s'en manifestent plus directement. Pourtant, la seconde ligne prend le pas sur la première : tout le monde se met à guetter les apparitions de la casaque rouge, dont le destin ponctue le déroulement de la fiction et qui intervient même au niveau de la première histoire, puisque quand ses pièces sont finalement réunies, les embûches qui empêchaient l'union des deux amants disparaissent comme par enchantement.

9 Mais de manière significative, le récit se termine sur la vision plus problématique et inquiétante d'une noce de village qui se transforme en « danse des morts » et donne lieu à une conclusion profondément élégiaque du narrateur :

Le spectateur aurait été saisi d'un sentiment étrange, inexprimable, en voyant comment un seul coup d'archet du musicien aux moustaches frisées, vêtu d'une 
casaque de coutil, avait suffi, bon gré mal gré, à mettre tout ce monde à l'unisson et à faire régner l'entente. Des gens dont les visages maussades n'avaient jamais, semble-t-il, été effleurés par le moindre sourire, battaient la mesure du talon et secouaient les épaules en cadence. Mais, plus étrange, plus indéfinissable encore le sentiment qui se serait éveillé au tréfonds de l'âme du spectateur à la vue des vieilles, dont les visages décrépits respiraient l'indifférence de la tombe et qui se pressaient dans la foule, mêlées à des hommes neufs, riants et vivants. Que d'insouciance! Sans l'ombre d'une joie, même enfantine, sans une étincelle de sympathie, elles que l'ivresse seule animait, comme un mécanicien son automate sans vie, leur faisant accomplir des gestes d'apparence humaine, elles dodelinaient doucement leurs têtes enivrées et dansaient avec la foule en liesse, sans même tourner les yeux vers les jeunes mariés. (Gogol, 1966, 46-47).

Cette fin plus sombre reproduit l'atmosphère folklorique du vertep, qui préside à la narration de Panko et se caractérise par le mélange du pittoresque paysan et du surnaturel, mais elle pose aussi des questions d'ordre narratif : qui parle ici? Si une puissance d'un autre monde (le diable) est aux commandes de cette action apparemment immanente (le mariage des deux jeunes paysans), ne faut-il pas comprendre que le conteur est lui-même doublé par une figure surplombante qui le domine?

Ces deux textes instaurent en effet une zone de flou énonciatif qui s'apparente à un «paradoxe de l'autorité narrative » :

La voix d'un conteur, voire d'un romancier qui écrit comme parle un conteur, est inscrite à l'origine d'un énoncé écrit : il y a paradoxe au sens où cette voix joue bien son rôle de justification de l'acte narratif, en plaçant à l'origine du récit un je fondé à narrer, mais brouille en même temps l'identité de ce sujet qui se donne à travers la double détermination de l'oral et de l'écrit, du conte et du roman. (Rabau, 2000, 399).

\section{Vers la production d'un narrateur : les indices d'une dissociation progressive d'avec le conteur}

Il semble bien y avoir deux voix à l'œuvre dans le système énonciatif des «veillées » : en effet, toute une série d'indices engage à effectuer une dissociation entre le conteur et le narrateur dans les textes, et à déceler en définitive une tension entre la visée réaliste de l'écriture ethnographique et les enjeux proprement esthétiques à l'œuvre, ainsi que l'a montré Jean-Marie Privat (2011). Autant le conteur est une figure fondamentale de la justification de l'œuvre, auquel il apporte son aura charismatique et sa légitimité documentaire, autant il apparaît assez rapidement comme un narrateur insuffisant pour prendre en charge le récit, et en particulier son écriture. Ainsi, nous nous interrogions sur la voix narrative à l'œuvre dans la précédente citation, qui conclut «la Foire de Sorotchintsy » sur une vision panoramique de la fête de village : or, il paraît clair qu'elle ne peut être celle de Panko le Rouge, narrateur terre-à-terre, dont le point de vue reste toujours limité puisque c'est son incapacité à intervenir sur les données de son récit qui en font un conteur fiable et donc intéressant.

Le long avant-propos à la première partie des Veillées du hameau souligne en effet à la fois le verbe pittoresque du narrateur et son point de vue restreint: dès le second paragraphe, Panko se décrit en humble paysan, issu du fin fond de la campagne (gluš), et qui s'attire le mépris de tous dès qu'il sort du périmètre de son modeste village $\left(x u t o{ }^{1}\right)$. De plus, sa propre parole est marquée par une verbosité ostentatoire, que lui- 
même reconnaît au terme de ce prologue: «mais je bavarde!» (Gogol, 1966, 16). D'ailleurs, ce jacasseur n'est souvent qu'un intermédiaire dans les histoires racontées dans le recueil : ce n'est pas toujours lui qui les profère, mais il les rend possibles en accueillant les narrateurs chez lui : "Mais nulle part, je crois bien, on n'a raconté autant de curiosités qu'aux soirées de l'apiculteur Panko le Rouge » (Gogol, 1966, 12). La parole à la source du récit est ainsi éclatée entre différents narrateurs anonymes, ainsi qu'en témoigne la tournure impersonnelle de la citation précédente: lorsque Panko entreprend de lire une histoire d'un narrateur individualisé, celui-ci lui signale qu'il ne s'agit pas de la sienne - la matière populaire apparaît bien ici comme un ensemble de textes aux contours flous, jamais répétés à l'identique et difficiles à assigner à un auteur singulier. Seules certaines de ces autres figures du récit sont présentées dans l'avant-propos, comme Foma Grigoriévtich, le sacristain de l'église de Dikanka, à qui Panko reprend deux histoires - mais on ne saura pas lesquelles, ce qui accentue l'effet de dépersonnalisation de la narration. Ce manque d'auctorialité est accentué par le fait que Panko encourage même le lecteur-auditeur à colporter lui-même des histoires sur la vie du hameau: "Venez seulement chez nous, venez vite, vous verrez comme nous vous ferons manger : vous irez ensuite le raconter à tout venant » (Gogol, 1966, 16).

Mais c'est surtout son incapacité constitutive à s'élever au-delà de la sphère du témoignage direct qui disqualifie Panko par rapport à une véritable figure de narrateur. La concurrence des instances narratives est directement représentée dès l'avantpropos : Panko y décrit en détail l'attitude d'un jeune homme au manteau couleur petit pois, qui est, comme on l'apprend au début de la seconde nouvelle, à l'origine de «la Foire de Sorotchintsy». Le conteur rapporte même les paroles de ce "narrateur subtil », renforçant le sentiment d'une authentique "polyphonie culturelle» (Privat, 2011):

Le narrateur subtil n'a pas trouvé l'histoire à son goût : sans dire un mot, il a quitté sa place, s'est campé, jambes écartées, au milieu de la pièce, a légèrement incliné la tête en avant, enfoncé la main dans la poche arrière de son caftan couleur de petit pois, en a tiré une tabatière de bois laqué, a fait claquer du doigt la trogne du général mécréant qui était dessinée dessus et, prenant une bonne portion de tabac pilé avec des cendres et des feuilles d'angélique, l'a balancée jusqu'à son nez, lequel a aspiré au vol la pincée entière, sans même effleurer le pouce, - et tout cela sans dire un mot ; puis, il a tiré d'une autre poche un mouchoir de coton bleu à carreaux, et c'est alors seulement qu'il a grommelé dans sa barbe un dicton, je crois bien: «Ne jetez pas de perles aux cochons ». (Gogol, 1966, 16).

La profusion de détails crée évidemment un effet comique, mais elle signale aussi que Panko ne parvient pas à adopter une attitude surplombante, et ne peut que décrire avec verve et fidélité les circonstances qu'il côtoie lui-même, sans percer leur mystère ni évoquer leurs raisons profondes, ce qui se traduit ici par des gesticulations opaques et des mots à demi-proférés qui ne seront jamais élucidés. Au contraire, le «narrateur subtil » est celui qui peut s'extraire de ce point de vue prosaïque et se disséminer dans l'ensemble de la fiction sur le mode d'un point de vue global : face au témoin Panko qui ne fait qu'exhiber la matérialité de sa collecte de faits et dont la personnalité fait obstacle à la pleine compréhension des événements, ce narrateur est subtil précisément parce qu'il est soluble. Il ne se parle pas directement dans la fiction: on le voit apparaître en marge de l'action et de l'énonciation, dans l'avant-propos, puis au début de la seconde nouvelle «la Veille d'Ivan Kupala », mais sur le mode de la mention, non parce qu'il prend la parole en son nom propre. Il faut donc le chercher dans le récit, contrairement à Panko : la quête que «la Foire de Sorotchintsy » instaure n'est pas 
seulement celle de la casaque rouge du diable, mais celle de ce caftan couleur petit pois, qui renvoie à Gogol lui-même, ainsi que le souligne Friedrik Driessen :

Il ne fait aucun doute qui Gogol entendait par ce jeune homme de la Poltava. L'homme qui se tient à part, pour qui ce monde joyeux n'est qu'un jeu dont il peut être le seul observateur, nostalgique et angoissé, cet homme qui n'est ni un moscal [Russe ethnique] ni un khokhol [Cosaque d'Ukraine], qui peut-il être d'autre que Gogol ? (Driessen, 1965, 71).

George Sand tient également à dissocier les deux voix présentes dans le texte : celle du conteur est une voix représentée comme réelle, et à ce titre elle possède toutes les limitations d'une voix véritable. Ainsi, le texte fait mention des bruits parasites qui recouvrent la voix du chanvreur, comme des limitations propres au corps qui la porte :

C'est durant ces nuits-là, nuits voilées et grisâtres, que le chanvreur raconte ses étranges aventures de follets et de lièvres blancs, d'âmes en peine et de sorciers transformés en loups, de sabbat au carrefour et de chouettes prophétesses au cimetière. Je me souviens d'avoir passé ainsi les premières heures de la nuit autour des broyes en mouvement, dont la percussion impitoyable, interrompant le récit du chanvreur à l'endroit le plus terrible, nous faisait passer un frisson glacé dans les veines. Et souvent aussi le bonhomme continuait à parler en broyant; et il y avait quatre à cinq mots perdus : mots effrayants, sans doute, que nous n'osions pas lui faire répéter, et dont l'omission ajoutait un mystère plus affreux aux mystères déjà si sombres de son histoire. (Sand, 1964, 139-140).

La présence de la machine, quoique discrète, n'est pas anodine. Elle accentue en effet le transfert de compétences auctoriales en substituant, à l'opposition intuitive de la fluidité immédiate de la parole et de la besogne seconde de l'écriture où c'est le premier terme qui est valorisé, la hiérarchie traditionnelle qui fait passer les artes liberales devant les artes mechanica: si ce sont des arts "libéraux", c'est bien parce qu'ils libèrent ceux qui les pratiquent de tout travail manuel au profit de l'activité de l'esprit, tandis que les arts mécaniques, dont le premier est le travail de la laine dans la définition canonique de Jean Scot Érigène, restent prisonniers des outils. La mention du bruit produit par le chanvrage met d'ailleurs en relief aussi bien les avantages (l'enargeia) que les inconvénients du récit oral : celui-ci, soumis aux circonstances de l'énonciation directe, peut être interrompu de manière arbitraire, notamment par la fatigue du narrateur souvent signalée dans le texte - ce qui fait ressortir par contraste la qualité décontextualisée du scriptural: "Savez-vous qu'il y a longtemps que je parle? dit-elle aux paroissiens qui l'écoutaient. Je n'ai plus le poumon comme à quinze ans. » (Sand, 1976, 113) dit Monique, la servante, avant de passer la main au chanvreur. Mais lui-même peut connaître ce type d'interruptions : "Mais voilà qu'il se fait tard, messieurs mes amis, et je m'endors sur mon histoire. À demain ; si vous voulez, je vous dirai le reste. Bonsoir la compagnie. » (Sand, 1976, 149).

Dans leur traitement particulier de la matière des "veillées ", destiné à introduire une dissociation entre la voix du conteur figuré et la voix narrative qui préside à l'organisation du texte, Sand et Gogol recourent à deux procédés identiques: ils finissent par exposer le subterfuge en donnant aux premières veillées une suite, dans laquelle le narrateur «subtil » est plus clairement désigné comme la véritable voix du récit, et en organisant le vieillissement prématuré de leur conteur au fil du récit.

Ainsi, le rôle dévolu au conteur des Veillées du hameau change au fil de l'œuvre, par l'adjonction d'un second livre de récits. Cette succession met en relief un déplacement : la seconde partie de l'œuvre se concentre sur le monde de la maison, plutôt que sur celui du xutor, le village ukrainien que le titre français le plus courant traduit par 
"hameau», et marque l'effacement du conteur exubérant au profit d'un discret narrateur de type omniscient. En effet, ces deux livres possèdent une organisation parfaitement structurée et se reflètent l'un dans l'autre: chaque livre est en effet organisé en quatre histoires, qui évoquent une saynète de guignol issue du vertep, une histoire inspirée par l'opéra comique, un récit sur les aïeux et un récit d'horreur. Cette symétrie souligne qu'une telle organisation ne peut être le fruit du hasard: la ligne souple des récits racontés à la veillée en fonction des personnes présentes et de l'humeur du conteur acquiert dans la suite du texte une vraie nécessité, qui montre que l'économie générale de l'œuvre n'est pas dictée par des circonstances extérieures au récit, mais par une volonté narrative interne à l'écriture du texte.

La disqualification progressive du conteur se manifeste corrélativement par le fait qu'il semble littéralement vieillir sous nos yeux dans le cours de la fiction, pour aboutir à une figure de vieillard impotent à l'orée de ce second livre où s'affirme le narrateur détaché, ainsi que le remarque Viktor Vinogradov:

Dans l'avant-propos à l'«autre livre » des Veillées, lors de leur seconde rencontre littéraire, il [Panko] s'adresse déjà au lecteur comme à une très ancienne connaissance. Le ton de la conversation se fait d'autant plus celui d'un ancêtre que le grand âge est l'un des thèmes de l'avant-propos. La sénilité rejaillit sur l'organisation stylistique du discours. Et par conséquent, le processus de décrépitude du vieillard Panko se produit durant la période de son accointance avec le lecteur. (Vinogradov, 1976, 264).

21 De manière significative, George Sand utilise la même stratégie que Gogol pour rendre sensible la séparation progressive des instances narratives. Son dernier roman champêtre, les Maîtres sonneurs (1853), constitue en effet une forme de suite au premier ensemble, dans laquelle on retrouve un chanvreur cette fois mieux identifié et dont l'existence remonte à un passé déjà ancien, là où le chanvreur du cycle originel semblait parfaitement contemporain du temps de la publication. Le texte radicalise ainsi l'imitation de la soirée paysanne déjà présente dans les Veillées du chanvreur: le chapitrage du roman est lui-même divisé en veillées, là où les romans précédents ne faisaient que suivre de manière plus ou moins lâche les interruptions supposées du chanvreur. Ce choix provoque une surdétermination du contage nocturne dans le texte : la veillée y apparaît à la fois comme le temps de la narration, le temps de l'action (l'histoire évoque les "cornemuseux" du Berry au xvIII siècle, musiciens qui se produisent en particulier la nuit, dans les auberges et les fêtes de village), et un élément constitutif de l'atmosphère, temps de l'amour entre les «jeunesses ${ }^{2}$ » des villages ennemis et de la confrontation avec des forces obscures. Le motif de la veillée est par ailleurs accentué par la présence d'un chanvreur bien identifié, Étienne Depardieu, qui se substitue à la figure idéalisée et anonyme des Veillées du chanvreur : le texte s'ouvre d'ailleurs sur une dédicace extérieure à la fiction, qui identifie les deux narrateurs, Sand et Depardieu, comme des personnes réelles et tend d'autant plus à avérer la situation de contage nocturne à l'origine du récit que la dédicace est signée " George Sand, Nohant, le 17 avril 1853 ", accentuant le processus d'identification :

Mon cher enfant, puisque tu aimes à m'entendre raconter ce que racontaient les paysans à la veillée, dans ma jeunesse, quand j'avais le temps de les écouter, je vais tâcher de me rappeler l'histoire d'Étienne Depardieu et d'en recoudre les fragments épars dans ma mémoire. Elle me fut dite par lui-même, en plusieurs soirées de breyage; c'est ainsi, tu le sais, qu'on appelle les heures assez avancées de la nuit où l'on broie le chanvre, et où chacun alors apportait sa chronique. (Sand, 1865, I). 
Pourtant, une telle série de superposition de motifs nocturnes jette le soupçon sur le caractère fortuit de cet agrégat: la surdétermination s'apparente en définitive à une « mise à nu du procédé » (Chklovski, 1973, 214), qui en révèle le caractère artificiel et faussement réalisant. L'aspect réaliste de la situation de contage s'efface par la distance temporelle que Sand souligne dans la préface et au début du texte prétendument raconté par Depardieu : le récit lui en a été fait il y a très longtemps, à l'époque où Depardieu était encore vivant, et le chanvreur lui-même relate une histoire qui a eu lieu dans sa prime jeunesse, au siècle passé. Son grand âge est par ailleurs accentué par un effet de coïncidence des dates : il dit être né en 1754, soit quasiment un siècle avant la parution du roman dans le Constitutionnel en 1853. Celui qui est présenté comme un conteur réel et identifié est donc d'emblée réduit à n'être qu'une figure stylisée, disparue depuis longtemps, et qui laisse ainsi le champ libre au narrateur véritable.

Ces effets de brouillage et de contradiction conduisent en effet à une interrogation : à quoi correspond le chapitrage du texte en veillées dans les Maîtres sonneurs? De fait, Sand ne s'explique jamais sur ce point : les divisions qu'elle introduit dans le texte sontelles censées respecter "les plusieurs soirées de breyage» (nous soulignons ce " plusieurs ») durant lesquelles Depardieu a raconté l'histoire? Le texte dément cette interprétation: d'abord, parce qu'il y a trente-deux veillées dans le texte, ce qui est trop pour recouvrir ce " plusieurs »; ensuite, parce qu'elles sont organisées en unités, non pas selon une division temporelle réaliste qui simulerait le temps de la narration (les chapitres sont en réalité d'inégale longueur, mais en suivant l'évolution de l'intrigue - ce que montre l'alternance du traitement des deux histoires d'amour présentes dans le texte, qui occupent une "veillée » à tour de rôle. Cela signifie que les « veillées » correspondent en réalité exactement à des chapitres, et que leur dimension pseudo-réaliste ne fait que renvoyer en définitive à leur textualité avérée : le lointain événement de contage nocturne qui justifie à l'origine le récit est désormais exposé comme une source insuffisante, et cette impossibilité même souligne le travail de recréation et de division de l'intrigue effectuée dans le cadre du texte lui-même. La "veillée » apparaît ici comme une fiction temporelle, affichée et justifiée dès le début du texte, mais servant en réalité de fausse amorce réalisante qui renvoie en définitive au livre lui-même.

24 La figure du conteur sert donc bien à légitimer le texte, mais elle est censée disparaître au fil des récits, au profit d'un narrateur détaché et surplombant dont la présence est d'autant plus frappante qu'il renvoie nettement, dans les deux textes étudiés, à l'auteur lui-même.

\section{La résolution de la concurrence des instances du récit au profit du narrateur : maîtrise focale et linguistique des narrateurs « subtils »}

Il y a donc bien une concurrence des instances narratives, puisque deux voix sont distinguées et que l'une est finalement valorisée par rapport à l'autre. Pourtant, cette disqualification progressive de la voix du conteur n'est pas agressive : le conteur reste un pivot de l'œuvre, mais il s'agit néanmoins pour les auteurs de suggérer qu'il ne saurait être seul tenu responsable de la production du texte et de l'organisation de la narration. Dans ce contexte, c'est bien une autre valeur de la représentation du contage 
qui se dessine: l'imaginaire de la veillée n'intervient pas uniquement comme un surplus de pittoresque, elle est bien un étai pour consolider par contraste une figure de narrateur qui se démarque du conteur en termes de focalisation et de langue.

On a dit que le cadre des Veillées du hameau désigne comme narrateur de la nouvelle étudiée une figure qui évoque beaucoup Gogol lui-même. Cette «manifestation" de l'auteur est ambiguë. Celui-ci intervient discrètement, en marge de la nouvelle, et ne prend pas directement la parole : à ce titre, comment se distingue-t-il du conteur, présenté comme péchant par son point de vue restreint? En réalité, Gogol ne peut être un personnage du livre que s'il est aussi l'auteur du livre maitrisant le déroulement de l'histoire et que si la fiction orale se délite, laissant apparaitre que Gogol, contre Panko le Rouge, est la seule source à l'origine du texte. On se retrouve dans une posture autocontradictoire : la situation orale révèle son impossibilité même, et souligne en réalité son essence scripturale. Ce glissement a deux conséquences majeures. D'une part, la surdétermination du narrateur Panko ne fait en définitive pas de lui le narrateur autoritaire que l'on pourrait croire, puisqu'il est discrètement discrédité, mais un personnage à qui l'on donne de la voix: son discours appartient donc au domaine du dialogue de personnage, et non de la voix autoritaire d'un narrateur qui donnerait le point de vue canonique sur la fiction. Ainsi, en dépit du fait que l'on se trouve dans un récit-cadre oral avec une narration à la première personne, on est bien dans une structure de showing (récit d'événements, selon la terminologie de Gérard Genette) et non de telling (récit de paroles). D'autre part, on observe une textualisation complète de la figure de l'auteur, qui apparaît comme un narrateur présent dans le récit non sur le mode de la voix corporelle, mais sur celui d'une voix narrative désincarnée et diffuse.

La fin élégiaque de l'histoire que nous citions au début de cet article suggère bien qu'il ne peut en définitive subsister qu'une seule des deux voix narratives : elle dessine la figure d'un individu solitaire qui seul subsiste quand l'animation de la foire s'est dissipée et qui seul peut jeter sur les événements un regard rétrospectif marqué par le sentiment du passage du temps. Cette posture en retrait justifie sa position dominante et le distingue profondément des autres instances narratives : il est comparé à un son unique qui continue de jouer quand toute la fanfare s'est tue, image de la voix narrative qui se conserve lorsque les autres ont été disqualifiées et que les circonstances temporelles et spatiales présentées comme consubstantielles au récit ont changé. Le narrateur apparaît donc comme le tiers absent, dont le point de vue supérieur et détaché des conditions directes de l'énonciation représentée est la véritable source de l'écriture. Ce point est décisif, car l'imaginaire de la veillée dans les textes est traditionnellement lu comme une manière d'identifier la source de la narration écrite à une situation de contage réel : en réalité, l'étude de ces textes montre qu'il peut aussi se doubler d'un processus de différenciation par rapport au(x) conteur(s), où le narrateur se retire des conditions directes de l'énonciation représentée au profit d'une externalité de point de vue et devient une instance supérieure de contrôle.

Un déplacement identique peut s'observer dans le texte de George Sand, qui rappelle celui de Gogol par le fait que le narrateur « subtil » est clairement associé à l'auteur, et non au conteur figuré. Mais la narratrice sandienne se différencie d'abord sur le plan de la langue, avant d'affirmer son point de vue surplombant et général sur les événements. Autour du texte des Veillées du chanvreur, on trouve en effet un premier cadre, qui met en scène un dialogue de Sand et d'un personnage nommé «R.»: ce cadre devrait 
marginaliser la figure auctoriale en lui conférant un simple rôle de témoin de récits que Sand se serait contentée de transcrire. Mais dès le début du texte, le rôle de la narratrice se trouve élargi grâce à la mention des enjeux linguistiques autour du contage. Dans un premier temps, la problématique de la langue permet la promotion de la narratrice comme traductrice, figure intermédiaire entre le parler paysan et les goûts du lectorat éduqué, plus proche de l'accroisseur de texte que du simple transcripteur qui se dissimule dans l'ombre des auctoritates. Ce rôle narratif plus conséquent lui est assigné par «R.», représenté dans l'avant-propos de François le Champi en train de lui demander de redire le conte qu'ils ont entendu la veille :

Tiens, commence, raconte-moi l'histoire du Champi, non pas telle que je l'ai entendue avec toi. C'était un chef-d'œuvre de narration pour nos esprits et pour nos oreilles du terroir. Mais raconte-la-moi comme si tu avais à ta droite un Parisien parlant la langue moderne, et à ta gauche un paysan devant lequel tu ne voudrais pas dire une phrase, un mot où il ne pourrait pas pénétrer. Ainsi tu dois parler clairement pour le Parisien, naïvement pour le paysan. (Sand, 1976, 52-53).

Ce passage métatextuel a pour vocation d'insister sur le travail de la langue effectué par Sand pour rendre ces histoires pourtant caractérisées comme " intraduisibles » dans la Mare au diable: "Ce chant n'est, à vrai dire, qu'une sorte de récitatif interrompu et repris à volonté. Sa forme irrégulière et ses intonations fausses selon les règles de l'art musical le rendent intraduisible" (Sand, 1964, 36). Il opère ainsi une première différenciation avec le narrateur oral en insistant sur l'activité de Sand, d'abord comme traductrice, mais aussi, à terme, comme narrateur détaché.

Dans un second temps, les préfaces sandiennes élaborent la référence discrète à une traduction de l'original paysan en manifestations claires de la recomposition auctoriale à l'œuvre dans le texte, au-delà du premier projet de la simple transcription. La préface de la Petite Fadette revient sur ce moment en y ajoutant une dimension importante: François le Champi n'a pas été que " raconté », l'histoire a aussi été écrite par Sand. Cette mention réaffirmée dans l'ouvrage suivant insiste sur la séparation progressive d'avec le modèle oral :

- Te souviens-tu, me dit-il, que nous passions ici, il y a un an, et que nous nous y sommes arrêtés tout un soir ? Car c'est ici que tu me racontas l'histoire du Champi, et que je te conseillai de l'écrire dans le style familier dont tu t'étais servi avec moi.

- Et que j'imitais de la manière de notre Chanvreur. (Sand, 1999, 19, nous soulignons).

31 Ainsi, le lecteur est convié dès l'orée du nouveau texte à guetter les moments de rupture et d'incohérence dans l'origine assignée à la parole narrative, là où se révèle la dissociation entre intention documentaire qui suppose l'effacement de l'auteur et authentique travail narratif de recréation. La matière de la Mare au diable est ainsi organisée de part et d'autre de cette ligne de faille. D'un côté, Sand endosse à nouveau le rôle de la traductrice, version améliorée du transcripteur, mais posture néanmoins indexée, au moins fictivement à une circonstance réelle (ici, le récit de Germain); de l'autre, elle prend bien garde de signaler que son récit s'émancipe de ces conditions premières et contraignantes :

Ici finit l'histoire du mariage de Germain, telle qu'il me l'a racontée lui-même, le fin laboureur qu'il est! Je te demande pardon, lecteur ami, de n'avoir pas su te la traduire mieux ; car c'est une véritable traduction qu'il faut au langage antique et naîf des paysans de la contrée que je chante (comme on disait jadis). Ces gens-là parlent trop français pour nous, et, depuis Rabelais et Montaigne, les progrès de la 
langue nous ont fait perdre bien des vieilles richesses. Il en est ainsi de tous les progrès, il faut en prendre son parti. Mais c'est encore un plaisir d'entendre ces idiotismes pittoresques régner sur le vieux terroir du centre de la France [...]. Certaines coutumes sont si étranges, si curieuses, que j'espère t'amuser encore un instant, cher lecteur, si tu permets que je te raconte en détail une noce de campagne, celle de Germain, par exemple, à laquelle j'eus le plaisir d'assister il y a quelques années. (Sand, 1964, 153).

La posture modeste ( $«$ je te demande pardon, lecteur ami, de n'avoir pas su te la traduire mieux») est ici contrebalancée par le reprise du mythe antique du poète inspiré (« je chante (comme on disait jadis) ») : cette actualisation champêtre du arma virumque cano établit une analogie avec la posture enthousiaste du poète traversé par la parole du dieu, de même que la traductrice donne de la voix aux histoires et aux langues qui ne sont pas les siennes, sans pour autant abdiquer son indépendance. L'intransivité du «je chante» est en effet confirmée par la suite de la citation, qui propose au lecteur un récit entièrement du cru de l'auteur, dans lequel la circonstance originelle se dissout : c'est d'« une noce de campagne » qu'il s'agit, et si l'on revient à celle de Germain, ce n'est qu'un « exemple » parmi d'autres; et c'est bien la narratrice elle-même, témoin des épousailles, qui sert cette fois de garant, sans l'intermédiaire du conteur.

La fin du dialogue avec « R. » cité plus haut suggère même que le chanvreur est relégué à un simple inspirateur, qu'il est là essentiellement pour suggérer un style narratif et non pour produire des histoires lui-même. Le titre des "veillées du chanvreur " semblait utiliser un génitif subjectif, désignant le chanvreur comme la source et le garant des récits : pourtant, il est dépossédé de ce statut par la promotion de la narratrice sandienne. Le lecteur s'en doutait ici encore, mais le texte prend soin de le dire : le titre n'est pas du chanvreur lui-même, et sa propre activité dans la gestation du texte est réduite à la portion congrue :

Je connais le chanvreur; il ne demande qu'à raconter des histoires, et il ne demeure pas loin d'ici. [...]

- Eh bien, allons le chercher, dit mon ami, tout réjoui d'avance; et demain tu écriras son récit pour faire suite, avec la Mare au Diable et François le Champi, à une série de contes villageois, que nous intitulerons classiquement les Veillées $d u$ Chanvreur.

- Et nous dédierons ce recueil à nos amis prisonniers... (Sand, 1999, 25).

La possession de l'œuvre a changé de mains : c'est à Sand et Rollinat que revient le droit de dédier le recueil, et ils ont l'intention de l'adresser aux prisonniers politiques $d u$ tournant conservateur d'après 1848 , c'est-à-dire à un monde radicalement éloigné du Berry et du premier cercle d'auditeurs du chanvreur.

Cette étude comparée fait donc ressortir le double bénéfice de l'inscription de l'oralité dans le texte écrit : non seulement, la figuration du contage confère à l'œuvre charisme et pittoresque, mais elle engage également une stratégie de dissociation qui aide à mettre en relief une figure de narrateur détaché et surplombant. Le travail sur l'imaginaire de la veillée joue ainsi sur la nature complexe du texte artistique que souligne Iouri Lotman :

La nature sémiotique du texte artistique est double: d'un côté, le texte fait semblant d'être une réalité indépendante de l'auteur, une chose parmi les choses du monde réel. De l'autre côté, il rappelle constamment qu'il est une création de quelqu'un et qu'il signifie quelque chose. (Lotman, 2004, 106). 
Les veillées figurées dans les textes instaurent en effet une porosité au monde des "choses», notamment parce qu'elles reprennent la justification de l'autorité par l'inclusion d'une parole extérieure et de documents issus de la réalité: l'oralité nocturne semble jouer ici le rôle d'un dispositif réalisant, qui met en scène la production de la fiction et donc tend à désigner sa propre essence comme réelle. Mais en même temps, cette mise en abyme de la production du texte est elle-même une fiction, ce que le texte dans son ensemble contribue à souligner, et désigne par conséquent la fiction insérée comme une œuvre radicalement autonome des circonstances apparentes à laquelle on voulait la réduire. Le jeu spéculaire entre cadre et fiction insérée permet donc de retourner le sens dans lequel s'établit cette « réflexion »: « les rapports entre les deux textes rappellent les reflets dans le miroir, mais ce qui semblait être un objet réel devient un reflet défiguré de l'objet qui semble lui-même être un reflet » (Lotman, 2004, 108). Ainsi, cette " autre voix de la littérature écrite ", où l'on paraît distinguer, dans les textes de Sand et Gogol, l'écho d'un contage réel, apparaît en définitive comme un objet construit et assignable à un narrateur détaché.

\section{BIBLIOGRAPHIE}

BÉNICHOU, Paul, 1988, les Mages romantiques, Paris, Gallimard.

BEIZER, Janet, 2004, Écoute le chant du labourage : chant et travail de l'écriture dans « les Veillées du chanvreur », Littérature, vol. 134, n 134, p. 95-110.

CHKLOVSKI, Viktor, 1973, Théorie de la prose, Lausanne, L’Âge d'homme [trad. G. Verret].

DIAZ, Jose Luis, 2007, l'Écrivain imaginaire : scénographies auctoriales à l'époque romantique, Paris, Honoré Champion.

DRIESSEN, Frederik, 1965, Gogol as a Short-Story Writer, La Hague, Paris, London, Mouton.

FANGER, Donald, 1979, The Creation of Nikolai Gogol, Cambridge, Belknap Press of Harvard University Press.

GOGOL, Nikolaï, 1966, les Soirées du hameau près de Dikanka, Euvres complètes [sous la direction de Gustave Aucouturier], Paris, Gallimard, « Bibliothèque de la Pléiade » [trad. M. Aucouturier].

KoFMAN, Sarah, 1984, Autobiogriffures du «Chat Murr » d'Hoffmann, Paris, Galilée, « Débats ».

LOTMAN, Iouri, 2004, l'Explosion et la Culture, Limoges, PULIM [trad. I. Merkoulova, révisée par J. Fontanille].

LUBIN, Georges, 1992, George Sand, chantre du Berry, in George Sand, Promenade dans le Berry. Mœurs, coutumes, légendes, Bruxelles, Éd. Complexe, coll. « Le Regard littéraire », nº 55. p. 9-26. PRIVAT, Jean-Marie, 2011, la Mare au diable ou comment « faire le populaire », in Daniel Fabre et Jean-Marie Privat (dir.), Savoirs romantiques : une naissance de l'ethnologie, Nancy, Presses universitaire de Nancy, p. 257-289. 
PUTNEY, Christopher, 1999, Russian Devils and Diabolic Conditionality in Nikolai Gogol's "Evenings on a Farm near Dikanka", New York, Peter Lang.

RABAU, Sophie, 1998, le Conteur, son auditeur et le texte. À propos de « Ceci n'est pas un conte » de Diderot, in Jean Bessière (éd.), Littérature et théorie : intentionnalité, décontextualisation, communication, Paris, Honoré Champion, p. 141-153.

RABAU, Sophie, 2000, Fictions de présence : la narration orale dans le texte romanesque du roman antique au $\mathrm{xx}^{\mathrm{e}}$ siècle, Paris, Honoré Champion.

SAND, George, 1865, les Maîtres sonneurs, Paris, Michel Lévy frères [1 ${ }^{\text {re }}$ éd., 1853].

SAND, George, 1964, la Mare au diable, Paris, Garnier-Flammarion [1 $1^{\text {re }}$ éd., 1846].

SAND, George, 1976, François le Champi, Paris, Gallimard [1 ${ }^{\text {re }}$ éd., 1848].

SAND, George, 1997, les Préfaces de George Sand, Édition établie et annotée par A. Szabó, Debrecen, Studia Romanica, 2 vol.

SAND, George, 1999, la Petite Fadette, Paris, Gallimard, « Le Livre de Poche » [1 ${ }^{\text {re }}$ éd., 1849].

SCHILLER, Friedrich, 1992, Die Götter Griechenlands (1793-1800), Schillers Werke, Nationalausgabe, t. 1 Gedichte: in der Reihenfolge ihres Erscheinens, 1776-1799, Weimar, Hermann Böhlaus Nachfolger [Erste Auflage, 1943].

SOMOFF, Victoria, 2007, From Authority to Author: Russian Orose on the Eve of the Novel, 1820-1850, Ph.D., Berkeley, University of California.

TERTZ, Abram [Siniavski, Andreï], 1978, Dans l'ombre de Gogol, Paris, Seuil [trad. G. Nivat].

VAILLANT, Alain, 2005, la Crise de la littérature : romantisme et modernité, Grenoble, ELLUG.

VAILLANT, Alain, 2010, Modernité, subjectivation littéraire et figure auctoriale, Romantisme, $\mathrm{n}^{\circ} 148,2$, p. 11-25.

WEBER, Max, 2005, le Métier et la Vocation de savant, le Savant et le Politique, Paris, Plon [ $1^{\text {re éd., }}$ 1963] [Trad. J. Freund].

\section{NOTES}

1. Le terme utilisé est délibérément marqué : il ne s'agit pas du terme générique pour désigner un village en russe, mais de celui qui s'applique spécifiquement à la campagne ukrainienne, ce qui accentue le caractère purement local du récit rapporté par Panko. De même, le terme de gluš utilisé pour décrire la région qu'habite Panko possède une connotation axiologique forte : il s'agit d'une campagne très éloignée et souvent peu civilisée.

2. C'est-à-dire des jeunes gens en patois : à part ce terme utilisé de manière systématique, le chanvreur que Sand figure dans le texte ne parle pas une langue très marquée par le patois nous reviendrons sur ce point dans notre dernière partie. 


\section{RÉSUMÉS}

L'article se propose d'étudier les processus de projection et de distanciation par rapport aux figures de conteur présentes dans les Veillées du hameau (1830-1843) de Nikolaï Gogol et les Veillées du chanvreur (1844-1849) de George Sand. Nous souhaiterions relire à l'aune du paradigme récent des «figurations auctoriales » ces deux ensembles de récits romantiques qui mettent au cœur de leur dispositif narratif la figuration d'un conteur paysan racontant des histoires lors de veillées : or, ces textes se caractérisent moins par une volonté d'imitation du récit folklorique que par un jeu des instances narratives qui permet de rapprocher, puis de dissocier conteur et narrateur. Grâce à la projection incomplète sur la figure du conteur, l'auteur peut ainsi à la fois s'inscrire dans une oralité imaginaire charismatique qui permet d'établir un contact direct avec son lecteur et rappeler en dernière instance qu'il dispose du monopole cognitif sur l'écriture de la fiction : l'inscription d'une «autre voix de la littérature écrite " lui a bien servi à affirmer une voix littéraire propre.

This paper analyses the processes of projection and distanciation towards the storyteller's figure in Evenings in a Farm near Dikanka (1830-1843) by Nikolaï Gogol and the story-cycle The Hemper's Watches (1844-1849) by George Sand. It seems interesting to read these texts in the perspective of the recent paradigm called "author figurations": these two sets of romantic stories use the narrative device of representing a peasant storyteller telling stories during evenings. Nevertheless, these texts are characterized, less by a desire to emulate the folktale, than by a wish to dissociate storyteller and narrator. With this incomplete projection on the figure of the storyteller, the author can thus both be part of a charismatic imaginary orality which allows direct contact with the reader and remind ultimately that he has the cognitive monopoly on writing fiction: this "other voice of written literature" has well served to affirm his own literary voice.

\section{INDEX}

Thèmes : littérature orale

Keywords : Storyteller, Narrator, Author Figuration, Sand George (1804-1876), Gogol Nikolaï (1809-1852)

Mots-clés : conteur, narration, figuration auctoriale, Sand George (1804-1876), Gogol Nikolaï (1809-1852)

\section{AUTEUR}

\section{VICTOIRE FEUILLEBOIS}

Université de Tours 